\title{
ALDH1A1 defines invasive cancer stem-like cells and predicts poor prognosis in patients with esophageal squamous cell carcinoma
}

Lang Yang ${ }^{1,8}$, Yong Ren ${ }^{1,8}, \mathrm{Xi} \mathrm{Yu}{ }^{1,8}$, Feng Qian ${ }^{2}$, Bai-Shi-Jiao Bian ${ }^{1,3}$, Hua-liang Xiao ${ }^{4}$, Wei-guang Wang ${ }^{5}$, Sen-lin $\mathrm{Xu}^{1}$, Jing Yang ${ }^{1}$, Wei Cui ${ }^{1}$, Qiang Liu ${ }^{1}$, Zhe Wang ${ }^{1}$, Wei Guo ${ }^{6}$, Gang Xiong ${ }^{7}$, Kang Yang ${ }^{7}$, Cheng Qian ${ }^{1}$, Xia Zhang ${ }^{1}$, Peng Zhang ${ }^{1}$, You-hong Cui $^{1}$ and Xiu-wu Bian ${ }^{1}$

${ }^{1}$ Institute of Pathology and Southwest Cancer Center, and Key Laboratory of Tumor Immunopathology of Ministry of Education of China, Southwest Hospital, Third Military Medical University, Chongqing, China; ${ }^{2}$ Department of General Surgery, Southwest Hospital, Third Military Medical University, Chongqing, China; ${ }^{3}$ Yongchuan Hospital, Chongqing Medical University, Chongqing, China; ${ }^{4}$ Department of Pathology, Daping Hospital, Third Military Medical University, Chongqing, China; ${ }^{5}$ Research Institute in Healthcare Science, University of Wolverhampton, Wolverhampton, UK; ${ }^{6}$ Department of Thoracic Surgery, Daping Hospital, Third Military Medical University, Chongqing, China and ${ }^{7}$ Department of Thoracic Surgery, Southwest Hospital, Third Military Medical University, Chongqing, China

Invasion and metastasis are the major cause of deaths in patients with esophageal cancer. In this study, we isolated cancer stem-like cells from an esophageal squamous cell carcinoma cell line EC109 based on aldehyde dehydrogenase 1A1 (ALDH1A1), and found that ALDH1A1 $1^{\text {high }}$ cells possessed the capacities of self-renewal, differentiation and tumor initiation, indications of stem cell properties. To support their stemness, ALDH1A1 high cells exhibited increased potential of invasion and metastasis as compared with ALDH1A1 low cells. ALDH1A1 ${ }^{\text {high }}$ esophageal squamous cell carcinoma cells expressed increased levels of mRNA for vimentin, matrix metalloproteinase 2, 7 and 9 (MMP2, MMP7 and MMP9), but decreased the level of E-cadherin mRNA, suggesting that epithelial-mesenchymal transition and secretary MMPs may be attributed to the high invasive and metastatic capabilities of ALDH1A1 ${ }^{\text {high }}$ cells. Furthermore, we examined esophageal squamous cell carcinoma specimens from 165 patients and found that ALDH1A1 high cells were associated with esophageal squamous dysplasia and the grades, differentiation and invasion depth, lymph node metastasis and UICC stage of esophageal squamous cell carcinoma, as well as poor prognosis of patients. Our results provide the strong evidence that ALDH1A $1^{\text {high }}$ cancer stem-like cells contribute to the invasion, metastasis and poor outcome of human esophageal squamous cell carcinoma.

Modern Pathology (2014) 27, 775-783; doi:10.1038/modpathol.2013.189; published online 8 November 2013

Keywords: aldehyde dehydrogenase 1A1; cancer stem-like cells; esophageal squamous carcinoma; invasion; metastasis; prognosis

Esophageal squamous cell carcinoma is one of the most frequent fatal malignancies in the area from northern Iran to north-central China ('Asian esopha-

Correspondence: Dr P Zhang, PhD, Dr Y-h Cui, MD or Dr X-w Bian, MD, PhD, Institute of Pathology and Southwest Cancer Center, and Key Laboratory of Tumor Immunopathology of Ministry of Education of China, Southwest Hospital, Third Military Medical University, Gaotanyan 30, Shapingba District, Chongqing 400038, China.

E-mail: p_zhangp@yahoo.com, youhongcuix@yahoo.com or bianxiuwu@263.net

${ }^{8}$ The first three authors contributed equally to this work.

Received 11 July 2013; revised 29 August 2013; accepted 1 September 2013; published online 8 November 2013 geal cancer belt'). ${ }^{1,2}$ The 5 -year survival rate of esophageal squamous cell carcinoma patients after surgery and chemotherapy remains low owing to highly invasive and metastatic nature of esophageal squamous cell carcinoma. Cancer stem-like cells are a small subpopulation within tumors with the capacities for self-renewal and generating heterogeneous tumor cell lineages. ${ }^{3}$ Recent studies suggest that cancer stem-like cells are responsible for invasion and metastasis of many tumor types. We have reported that cancer stem-like cells possess higher capability of invasion and metastasis in solid tumors. $^{4-6}$ However, the biomarkers related to invasion and metastasis of cancer stem-like cells 
and the clinical significance need to be identified in esophageal squamous cell carcinoma.

Aldehyde dehydrogenase 1A1 (ALDH1A1) is a cancer stem-like cell-associated protein in various malignant tumors and its level correlates with the patient outcome. ${ }^{7-9}$ We hypothesized that ALDH1A1 could also be used to detect and enrich cancer stemlike cells, and the level of ALDH1A1 ${ }^{\text {high }}$ cells in tumor might predict the prognosis of human esophageal squamous cell carcinoma. In this study, we report that the level of ALDH1A1-expressing cells in tumor correlates with the clinic outcome of esophageal squamous cell carcinoma patients. In addition, ALDH1A1 $1^{\text {high }}$ cells isolated from a human esophageal squamous cell carcinoma cell line exhibited properties of high invasiveness and metastasis mediated by epithelialmesenchymal transition and MMPs.

\section{Materials and methods}

\section{Patients and Tissue Samples}

Esophageal squamous cell carcinoma specimens used in this study were taken from 165 cases of esophageal squamous cell carcinoma patients with written informed consent from 2006 to 2008 at Southwest Hospital. No preoperative radiotherapy or chemotherapy was used for any of the patients. All the specimens were routinely processed for pathological diagnosis according to the WHO classification. The study was approved by the Ethics Committee of the Southwest Hospital.

\section{Immunohistochemistry}

Immunohistochemical staining was performed on formalin-fixed, paraffin-embedded esophageal cancer sections $(4 \mu \mathrm{m})$ using Dako REAL ${ }^{\mathrm{TM}}$ EnVision $^{\mathrm{TM}}$ detection System (Code K5007; Dako, Glostrup, Denmark). The sections were pretreated by $0.3 \%$ $\mathrm{H}_{2} \mathrm{O}_{2}$ and antigen retrieval was performed according to the manufacturer's instruction. The slides were incubated with mouse anti-human ALDH1A1 (Clone 44/ALDH, 1:1000; BD Bioscience, USA) at $4{ }^{\circ} \mathrm{C}$ overnight. The secondary antibody was added for incubation at $37^{\circ} \mathrm{C}$ for $30 \mathrm{~min}$. All slides were evaluated independently by two pathologists in a blind manner. The following points were taken as the guideline for interpreting the data: ${ }^{10}$ the sections with $>5 \%$ ALDH1A1-positive cancer cells as ALDH1A1 $1^{\text {high }}$ and $<5 \%$ positive cells as ALDH1A1 1ow. Briefly, the cells with brown color in the cytoplasm were counted as positive cells. At least five fields in each section were randomly selected for calculating average percentage of positive cells over total cancer cells. For the analysis of the relationship between esophageal squamous cell carcinoma invasiveness and distribution of ALDH1A1-positive cancer cells, the ALDH1A1-positive cells within the mucosa and invading into the submucosa were counted and their percentages were calculated.

\section{Cell Culture}

The human esophageal squamous cell carcinoma cell line EC109 was purchased from Cell Bank of Shanghai Institute of Cell Biology, Chinese Academy of Sciences, and cultured in Dulbecco's modified Eagle's medium (Gibco, USA) containing 10\% fetal bovine serum at $37^{\circ} \mathrm{C}$ in humidified air with $5 \% \mathrm{CO}_{2}$.

\section{ALDEFLUOR Assay and Flow Cytometry}

An ALDEFLUOR kit (Stem Cell Technologies, Canada) was used to isolate tumor cells with ALDH1A1A1 enzymatic activity according to the manufacturer's instruction. Briefly, esophageal squamous cell carcinoma cells were suspended in ALDEFLUOR assay buffer containing ALDH1A1 substrate, add BODIPYaminoacetaldehyde (BAAA) and incubated for $40 \mathrm{~min}$ at $37^{\circ} \mathrm{C}$. Diethylaminobenzaldehyde (DEAB), a specific ALDH1A1 inhibitor, was used as a negative control. 7-Amino-actinomycin D (7-AAD) staining solution (BD Pharmingen, USA) was used to exclude dead cells. Cells with intact plasma membrane were sorted for experiments.

\section{Colony Formation Assay}

One hundred viable esophageal squamous cell carcinoma cells per well were seeded in each well of 24-well plates and cultured in Dulbecco's modified Eagle's medium containing 10\% fetal bovine serum. After incubation for 2 weeks, colonies containing more than 50 cells were counted with crystal violet staining.

\section{Limiting Dilution Assay}

Limiting dilution assay was used to measure the frequency of cancer stem-like cells as described. ${ }^{11}$ Briefly, six serial twofold dilutions of viable ALDH1A1A $1^{\text {high }}$ and ALDH1A $1^{\text {low }}$ cells were sorted into ultra-low 96-well plates (Costar, USA) with 10 wells per dilution. Sorted cells were cultured in $100 \mu \mathrm{l}$ of serum-free Dulbecco's modified Eagle's medium/F12 medium supplemented with EGF (20 ng/ml) and bFGF (20 ng/ml) at $37{ }^{\circ} \mathrm{C}$ and $5 \%$ $\mathrm{CO}_{2}$. Twenty microliters of serum-free Dulbecco's modified Eagle's medium/F12 medium supplemented with EGF $(20 \mathrm{ng} / \mathrm{ml})$ and bFGF $(20 \mathrm{ng} / \mathrm{ml})$ were added to each well every 3 days. Spheres were counted at day 7 .

\section{Immunofluorescence Confocal Microscopy}

For cell differentiation, FACS-sorted ALDH1A1 high cells were seeded on the cover slides and cultured in Dulbecco's modified Eagle's medium containing $10 \%$ fetal bovine serum for 7 days. The cells were then fixed and blocked by preimmune goat serum. 
Primary mouse anti-human CK18 (1:100) (Zhong Shan Jin Qiao Company, China) was added to the cells and incubated at $4{ }^{\circ} \mathrm{C}$ overnight. Secondary goat anti-mouse IgG conjugated with Cy5 (1:500) (Zhong Shan Jin Qiao Company) was added to the cells and incubated at $37^{\circ} \mathrm{C}$ for $30 \mathrm{~min}$. DAPI was used to stain the nuclei. Cells were then observed under laser confocal microscopy (Zeiss, Germany).

\section{RNA Extraction and Real-Time PCR}

Total RNA was extracted from tumor cells with RNAiso reagent (TakaRa, Japan). Reverse-transcription and quantitative real-time PCR were performed using an RNA PCR (AMV) kit (Fermentas, Canada) with primers designed for the following corresponding genes: Oct-4 (NM_001173531) (5'-GCAGCGAC TATGCACAACGA-3' (forward) and 5'-CCAGAGTGG TGACGGAGACA-3' (reverse)); Sox-2 (NM_003106) (5'-CATCACCCACAGCAAATGACA-3' (forward) and 5'-GCTCCTACCGTACCACTAGAACTT-3' (reverse)); Bmi-1 (NM_005180): (5'-TCGTTCTTGTTATTACGCT GTTTT-3' (forward) and 5'-CGGTAGTACCCGC TTTTAGGC-3' (reverse)); vimentin (NM_003380200) (5'-GGGACCTCTACGAGGAGGAG-3' (forward) and 5'-CGCATTGTCAACATCCTGTC-3' (reverse)); $C D H 1$ (NM_004360) (5'-TGCCCAGAAAATGAAAAAGG-3' (forward) and 5'-GTGTATGTGGCAATGCGTTC-3' (reverse)); MMP2 (NM_004530) (5'-CCACTGCCTTCG ATACAC-3' (forward) and 5'-GAGCCACTCTCTG GAATCTTAAA-3' (reverse)); MMP7 (NM_002423) (5'-GAGTGAGCTACAGTGGGAACA-3' (forward) and 5'-CTATGACGCGGGAGTTTAACAT-3' (reverse)); MMP9 (NM_004994) (5'-GTTCCCGGAGTGAGTT GA-3' (forward) and 5'-TTTACATGGCACTGC CAAAGC-3' (reverse)); and GAPDH (NM_002046) (5'-AGCCACATCGCTCAGACA-3' (forward) and 5'-G CCCAATACGACCAAATCC-3' (reverse)). The expression of each gene by $\mathrm{ALDH} 1 \mathrm{~A} 11^{\text {high }}$ relative to ALDH1A1 ${ }^{\text {low }}$ esophageal squamous cell carcinoma cells was determined using the $2-\Delta \Delta \mathrm{CT}$ method. Results were normalized against GAPDH. All experiments were performed in triplicates, and results were plotted as the mean \pm s.d.

\section{Invasion Assay}

Transwells $(8 \mu \mathrm{m})$ (BD Falcon, USA) were coated with $10 \mu \mathrm{l}$ of Matrigel ${ }^{\mathrm{TM}} /$ Dulbecco's modified Eagle's medium $(1: 6, \mathrm{v} / \mathrm{v})$ for $30 \mathrm{~min}$ at $37^{\circ} \mathrm{C}$. ALD$\mathrm{H} 1 \mathrm{~A} 1^{\text {high }}$ and ALDH1A1 $1^{\text {low }}$ esophageal squamous cell carcinoma cells were seeded into the upper Transwell chambers at the density of $5 \times 10^{4}$ in $200 \mu \mathrm{l}$ of serum-free Dulbecco's modified Eagle's medium. The lower chambers were filled with $500 \mu \mathrm{l}$ of Dulbecco's modified Eagle's medium containing $10 \%$ fetal bovine serum. After $24 \mathrm{~h}$ incubation, filter membranes were fixed with $4 \%$ formaldehyde for $10 \mathrm{~min}$. Subsequently, cells on the upper chamber were scraped by cotton swab, and the invading cells on the lower chambers were stained with crystal violet for $5 \mathrm{~min}$. The invading cells were counted in five different visual areas at $\times 100$ magnification under an inverted microscope.

\section{Tumorigenicity Assay}

Esophageal squamous cell carcinoma cells in Matrigel $^{\mathrm{TM}}(1: 1, \mathrm{v} / \mathrm{v})$ were injected subcutaneously into the axilla of 6 -week-old nude mice with $5 \times 10^{2}$ or $1 \times 10^{2}$ cells per mouse $(n=5)$. At the end of sixth week, mice were euthanized and xenograft tumors were removed and measured. Tumor volume $\left(T_{\mathrm{V}}\right)$ was calculated by the formula: $T_{\mathrm{V}}=L$ (length) $\times W^{2}$ (width) $/ 2$.

\section{In Vivo Metastasis Assay}

Intravenous injection was used to evaluate the lung metastasis ability of ALDH1A $11^{\text {high }}$ and ALDH1A $11^{\text {low }}$ esophageal squamous cell carcinoma cells as described. $^{12}$ Briefly, $1 \times 10^{5}$ sorted ALDH1A1 $1^{\text {high }}$ and ALDH1A $1^{\text {low }}$ cells were injected into the tail vein of 6 -week-old nude mice $(n=6)$. All mice were killed at the sixth week and examined for tumor nodules in the lung.

\section{Statistical Analysis}

Positive staining rates were compared by using $\chi^{2}$ method. Rank data was analyzed by Spearman's rank correlation test. Kaplan-Meier survival plots and log-rank statistics were used to compare the survival rates of patients. All data were analyzed with SPSS13.0 statistical software. $P<0.05$ was considered statistically significant.

\section{Results}

ALDH1A1 ${ }^{\text {high }}$ Esophageal Squamous Cell Carcinoma Cells Exhibit Cancer Stem-Like Cell Properties

We first examined the proportion of ALDH1A1 high cells contained in the esophageal squamous cell carcinoma cell line EC109, and found that ALDH1A $1^{\text {high }}$ cells accounted for $3.4 \% \pm 0.5 \%$ of the whole-cell population $(n=17)$ (Figure 1a). The sorted ALDH1A $1{ }^{\text {high }}$ cells possessed higher capabilities of tumorsphere formation as compared with ALDH1A $1^{\text {low }}$ cells (Figure 1b). The number of colonies formed by ALDH1A1 $1^{\text {high }}$ cells were higher than those formed by ALDH1A $1{ }^{\text {low }}$ cells $(76.6 \% \pm$ $1.5 \%$ vs $31.3 \% \pm 2.1 \%, P<0.05$ ) (Figures $1 \mathrm{c}$ and $\mathrm{d}$ ). The ALDH1A1 $1^{\text {high }}$ cells also showed increased expression of the stemness genes Sox2 and Bmi1 (Figure 1e) and high differentiation potential by expressing CK18 (Figure 1f). The tumorigenicity assay with ALDH1A1high and ALDH1A1 low EC109 cells in nude mice showed that $1 \times 10^{2} \mathrm{ALDH} 1 \mathrm{~A} 11^{\text {high }}$ cells initiated tumors in four of five mice $(80 \%)$. 
a

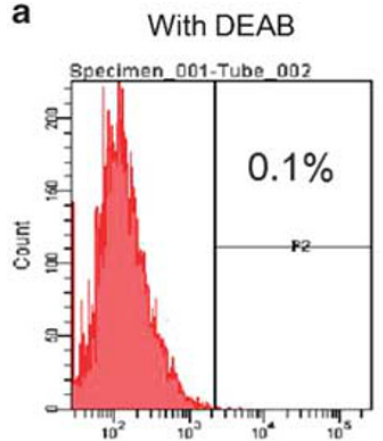

C

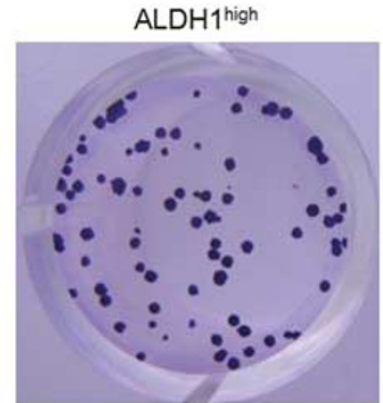

Without DEAB
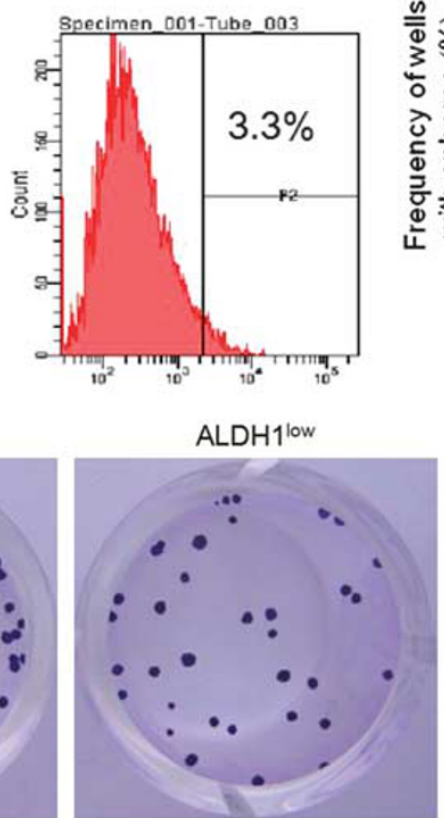
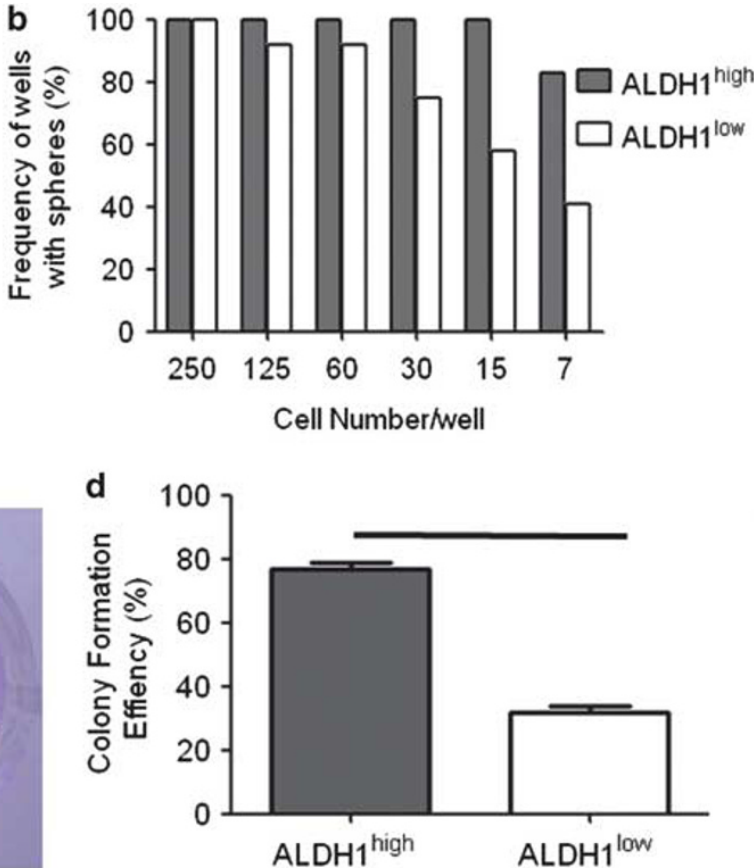

e

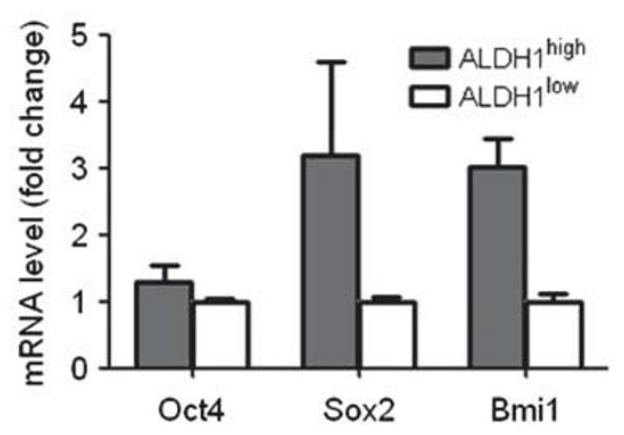

f

Induced differentiation
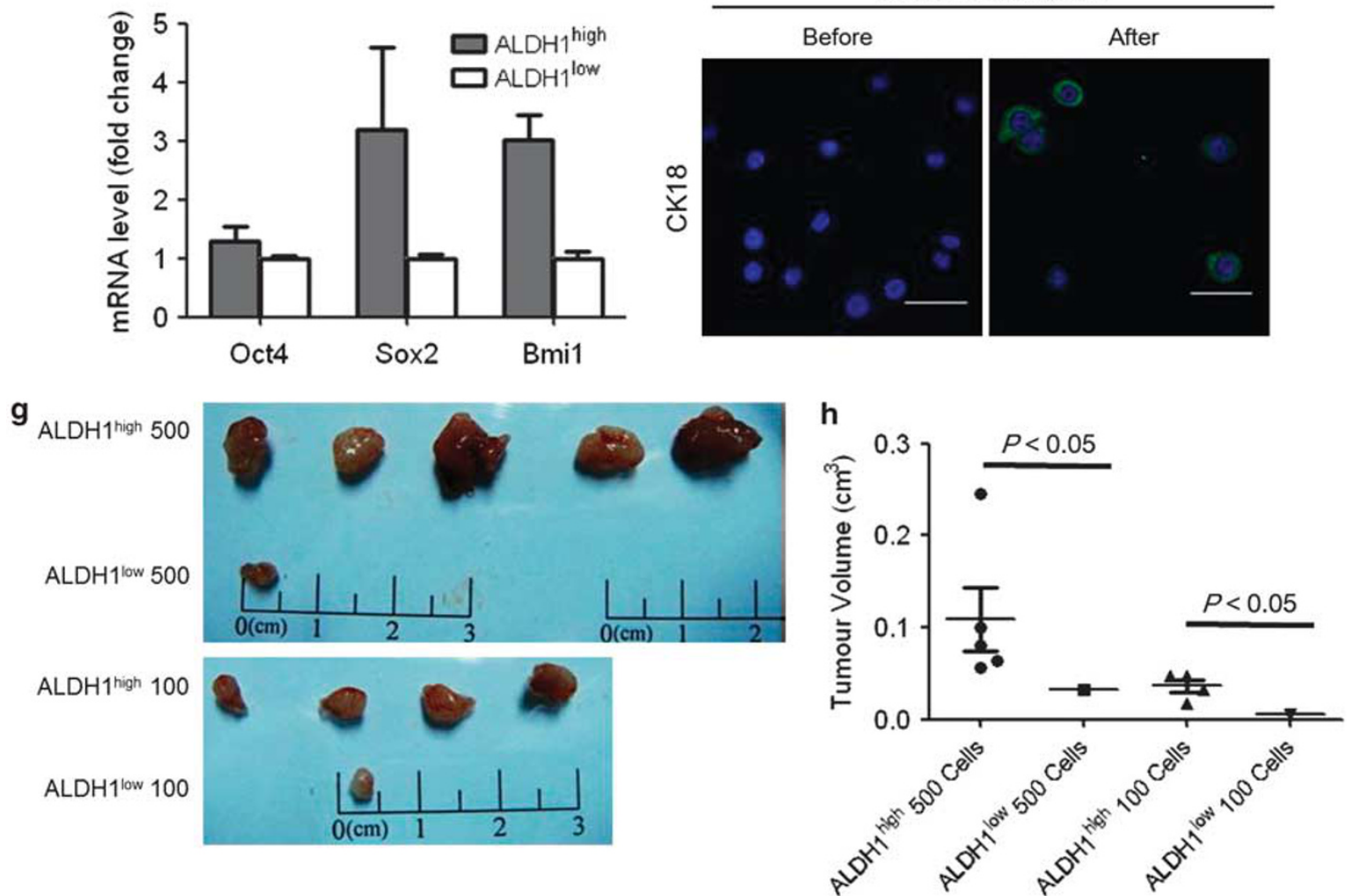

Figure 1 Characterization of ALDH1A1 ${ }^{\text {high }}$ cells. (a) Determination of the frequency of ALDH1A1 ${ }^{\text {high }}$ cells in EC109 cell line by flow cytometry. (b) Limiting dilution assay shows the self-renewal capability of ALDH1A1 high cells in vitro. (c) Colony formation of FACSsorted ALDH1A1 $1^{\text {high }}$ and ALDH1A $1^{\text {low }}$ cells. (d) Quantitative analysis of colony formation of FACS-sorted ALDH1A1 high and ALDH1A1 $1^{\text {low }}$ cells. (e) Measurement of Bmi1, Sox2 and Oct4 mRNA levels in ALDH1A1 high and ALDH1A1 ${ }^{\text {low }}$ cells by quantitative realtime polymerase chain reaction (qRT-PCR). (f) Detection of CK18 by immunofluorescence confocal microscopy showing the differentiation potential of ALDH1A $1^{\text {high }}$ cells. (g) Representative images of xenografted tumors formed by ALDH1A1high and ALDH1A1 1ow cells in nude mice. (h) Quantitative analysis of the volumes of xenografted tumors formed by ALDH1A1 ${ }^{\text {high }}$ and ALDH1A1 $1^{\text {low }}$ cells in nude mice. ALDH1A1, aldehyde dehydrogenase 1A1; FACS, fluorescence-activated cell sorter. 
All five mice $(100 \%)$ grew tumors when $5 \times 10^{2}$ ALDH1A $1^{\text {high }}$ cells were implanted. In contrast, implantation of $1 \times 10^{2}$ or $5 \times 10^{2}$ ALDH1A $1^{\text {low }}$ EC109 cells formed tumors only in one of five mice $(20 \%)$ (Table 1). The size of the xenograft tumors formed by ALDH1A $1^{\text {high }}$ cells was much larger than those formed by ALDH1A1 $1^{\text {low }}$ cells (Figures $1 \mathrm{~g}$ and $\mathrm{h}$ ).

\section{ALDH1A1 ${ }^{\text {high }}$ Cells Possess Highly Invasive and Metastatic Capabilities with Epithelial-Mesenchymal Transition Phenotype}

As invasion and metastasis are important features of esophageal squamous cell carcinoma, ${ }^{13,14}$ we examined the invasive and metastatic abilities of

Table 1 Tumorigenicity of ALDH1A $1^{\text {high }}$ and ALDH1A $1^{\text {low }}$ cells from the esophageal squamous cell carcinoma cell line EC109

\begin{tabular}{lcc}
\hline & \multicolumn{2}{c}{$\begin{array}{c}\text { Tumor } \\
\text { number/injected sites }\end{array}$} \\
\cline { 2 - 3 } Number of cells injected & ALDH1A1 $1^{\text {high }}$ & ALDH1A1 low \\
\hline $5 \times 10^{2}$ & $5 / 5$ & $1 / 5$ \\
$1 \times 10^{2}$ & $4 / 5$ & $1 / 5$ \\
\hline
\end{tabular}

the ALDH1A1 ${ }^{\text {high }}$ EC109 cells and found that such cells had a greater invasiveness as compared with ALDH1A1 1ow cells $(92.0 \pm 8.2$ vs $7.3 \pm 1.5, P<0.05)$ (Figures 2a and b). Moreover, the ALDH1A1 ${ }^{\text {high }}$ cells formed lung metastasis in five of six mice, whereas no metastasis was formed by the ALDH1A $1^{\text {low }}$ cells (Figure 2c). Similar results were also obtained from the studies on another esophageal squamous cell carcinoma cell line TE-1 (Supplementary Figure S1). We found that the percentage of ALDH1A1-positive cells was $8.3 \%$ in TE-1 (Supplementary Figure S1A) and sorted ALDH1A1 ${ }^{\text {high }}$ TE-1 cells possessed higher capabilities of sphere formation (Supplementary Figure S1B) and colony formation as compared with ALDH1A1 1ow cells $(72 \pm 8$ vs $25 \pm 5, \quad P<0.05)$ (Supplementary Figures S1C and S1D). In addition, ALDH1A1 1 high TE-1 cells were found with greater invasive ability as compared with ALDH1A1 low cells $(200 \pm 8$ vs $47 \pm 6, P<0.05)$ (Supplementary Figures $\mathrm{S} 1 \mathrm{E}$ and $\mathrm{S} 1 \mathrm{~F})$. Gene expression analysis further showed that ALDH1A1 high cells expressed higher levels of MMP2, MMP7, MMP9 and vimentin, and lower level of E-cadherin (Figure 2d), indicating that their highly invasive and metastatic capabilities are associated with an epithelialmesenchymal transition phenotype.

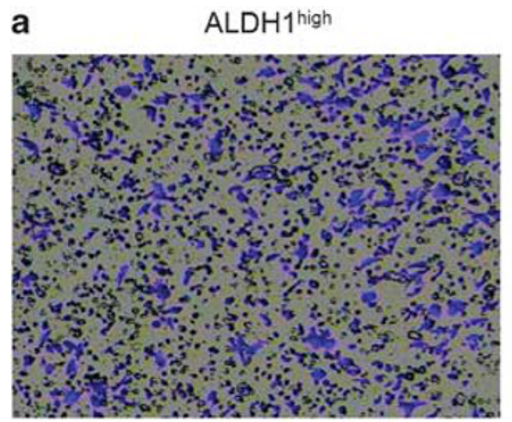

C
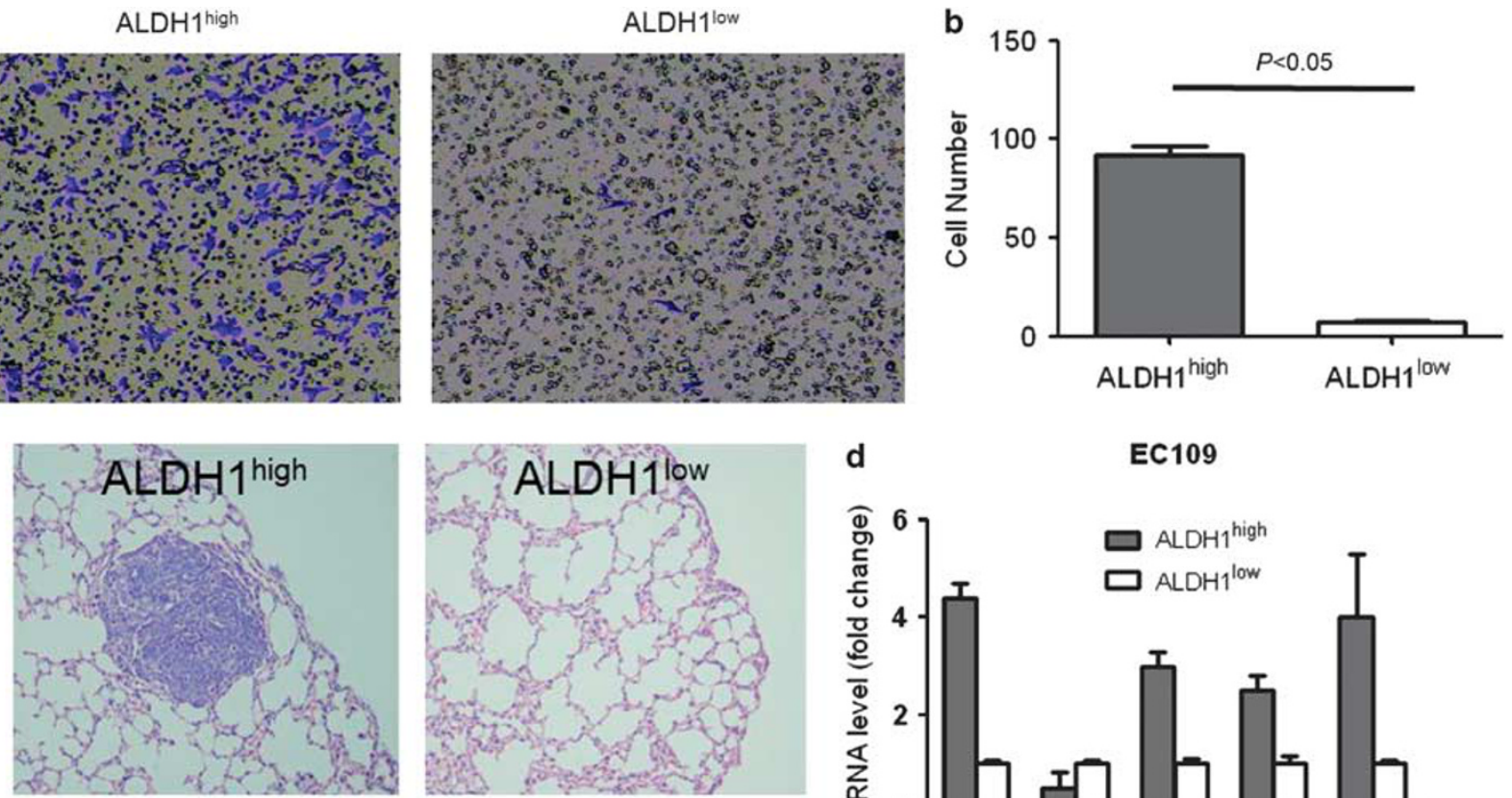

d

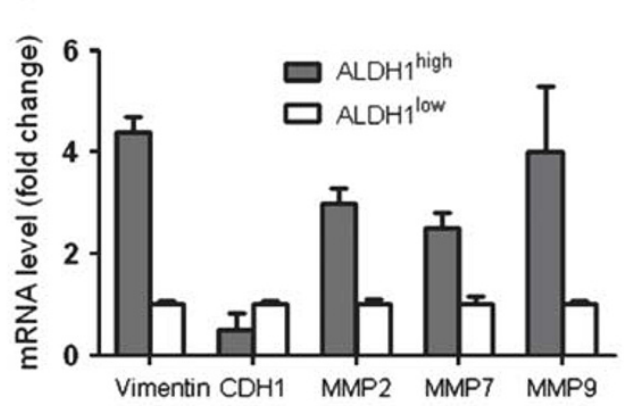

\begin{tabular}{cc}
\hline lung metastasis & (yes/no) \\
\hline ALDH1 $1^{\text {high }}$ & $5 / 1$ \\
ALDH1 1 low & $0 / 6$
\end{tabular}

Figure 2 Analysis of invasion and metastasis of ALDH1A1 $1^{\text {high }}$ cells and the expression of invasion-associated genes by ALDH1A1 ${ }^{\text {high }}$ cells. (a) In vitro invasion images of Transwell assay show increased number of ALDH1A1 high cells. (b) Quantitative analysis shows significant difference between invasion by ALDH1A $1^{\text {high }}$ and that by ALDH1A1 ${ }^{\text {low }}$ cells. (c) Enhanced lung metastasis of ALDH1A1 high cells injected into nude mice. (d) Quantitative real-time polymerase chain reaction (qRT-PCR) shows high levels of vimentin, MMP2, MMP7 and MMP9 expressions and low level of E-cadherin (CDH1) expression in ALDH1A1 ${ }^{\text {high }}$ compared with those in ALDH1A1 low cells. ALDH1A1, aldehyde dehydrogenase 1A1; MMP, matrix metalloproteinase. 


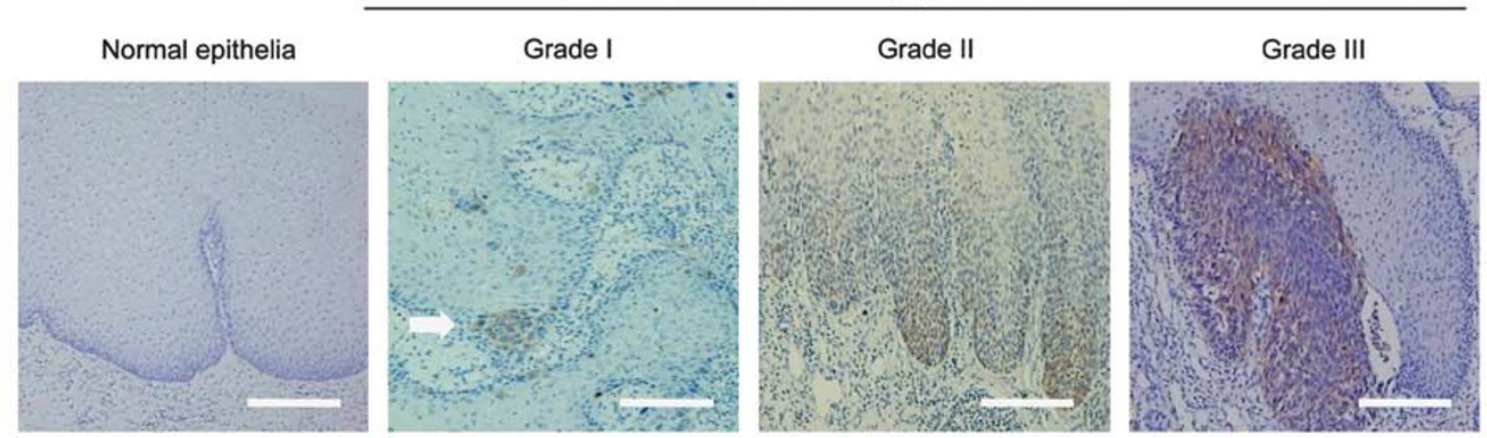

b

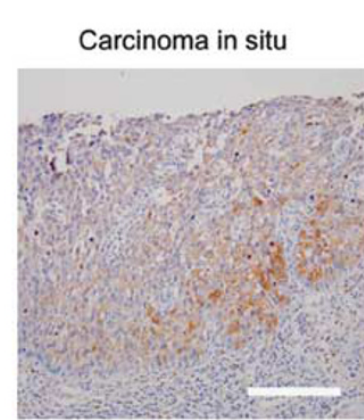

Early invasive

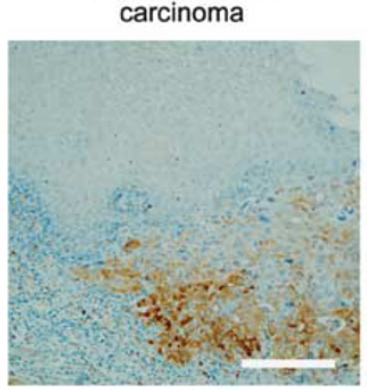

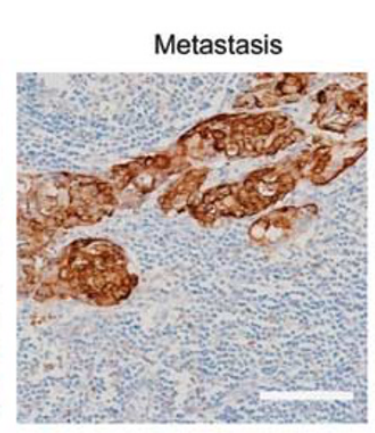

d

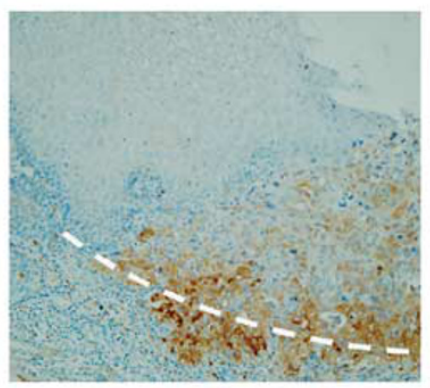

C

Tumor differentiation (grade)
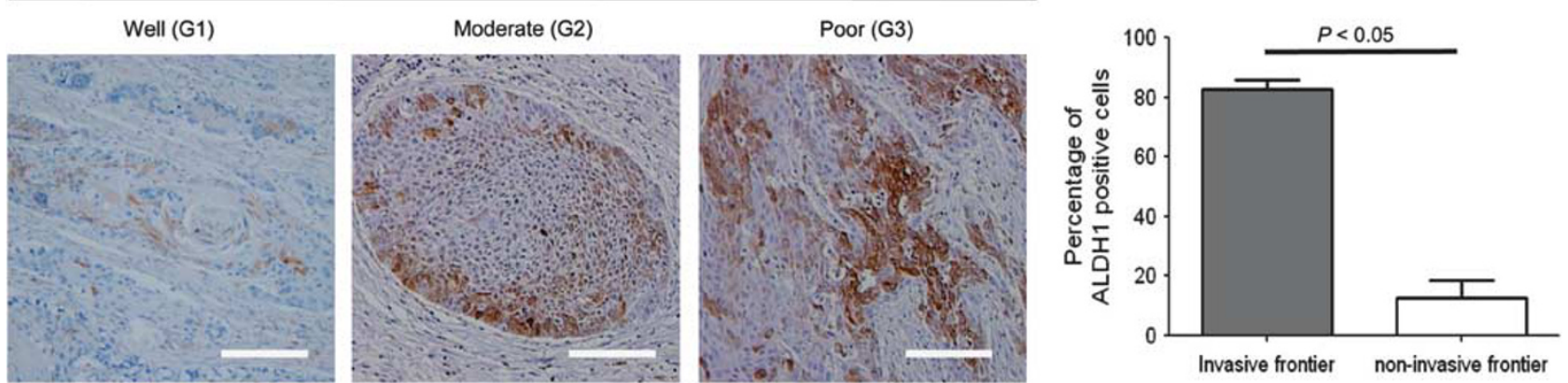

Figure 3 Immunohistochemical analysis of ALDH1A1-positive cells in normal esophageal mucosa and esophageal squamous cell carcinoma samples. (a) No ALDH1A1-positive cells are seen in normal esophageal mucosa, but ALDH1A1-positive cells are found increased in epithelial dysplasia. (b) Representative immunohistochemical images show the presence of ALDH1A1-positive cells in carcinoma in situ, early invasive carcinoma and lymph node metastasis, respectively. (c) Representative immunohistochemical images show enhancement of ALDH1A1-positive cells in esophageal squamous cell carcinoma along with the increases of histological grade. (d) Quantitative analysis of ALDH1A1-positive cells in submucosa and mucosa at T1 stage indicates more ALDH1A1-positive cells in the invasive frontier. An extending line from the epithelial basement membrane in the normal mucosa was drawn to distinguish the invasive frontier area and non-invasive frontier area (upper). Then, the ALDH1A1-positive cells and total cancer cells under the extending line (within submucosa) and those located above the extending line (within mucosa) were counted and their percentages were calculated (bottom). ALDH1A1, aldehyde dehydrogenase 1A1.

\section{ALDH1A1 Expression is Correlated with Esophageal Squamous Dysplasia and Carcinoma Malignancy}

For understanding the possible role of ALDH1A1 in the development and progression of human esophageal squamous cell carcinoma, we examined the expression of this protein in normal, dysplastic and cancerous esophageal epithelia. ALDH1A1 was not detected in normal esophageal epithelia, but was detectable at low levels in the dysplastic basal cells. The cytoplasmic ALDH1A1 was elevated in esophageal cells with increasing degrees of dysplasia and in carcinoma in situ (Figures 3a and b). Sixty-seven percent (110/165 cases) of esophageal squamous cell carcinoma samples were ALDH1A1 $1^{\text {high }}$ in primary tumors (Table 2). The expression of ALDH1A1 was positively correlated with histological grades $(P=0.004)$, invasion depth $(P=0.000)$, lymph node metastasis $(P=0.002)$ and UICC staging $(P=0.000)$ of esophageal squamous cell carcinoma (Table 2, Figure 3c). To reveal the relationship of ALDH1A1 expression cells to cancer cell invasiveness, we quantitatively analyzed ALDH1A1-positive cells in the tumor tissues of T1 stage esophageal squamous cell carcinoma. The percentage of ALDH1A1-positive cancer cells was significantly higher in tumor tissues invading submucosa than tumor tissues within the mucosa $(83 \% \pm 6 \%$ vs $13 \% \pm 12 \%$, 
Table 2 Relationship between ALDH1A1 and clinicopathological characteristics of esophageal squamous cell carcinoma

\begin{tabular}{|c|c|c|c|c|}
\hline \multirow[b]{2}{*}{ Clinical factors } & \multirow[b]{2}{*}{ Cases } & \multicolumn{3}{|c|}{$\begin{array}{c}\text { Esophageal squamous } \\
\text { cell carcinoma }\end{array}$} \\
\hline & & ALDH1A1 $1^{\text {low }}$ & $A L D H 1 A 1^{\text {high }}$ & $\mathrm{P}$-value \\
\hline \multicolumn{5}{|l|}{ Age (years) } \\
\hline$<60$ & 77 & 21 (27) & 56 (73) & 0.122 \\
\hline$\geq 60$ & 88 & $34(39)$ & $54(61)$ & \\
\hline \multicolumn{5}{|l|}{ Gender } \\
\hline Male & 137 & $49(36)$ & $88(64)$ & 0.158 \\
\hline Female & 28 & $14(50)$ & $14(50)$ & \\
\hline \multicolumn{5}{|c|}{ Histological grade } \\
\hline G1 & 91 & $39(43)$ & $52(57)$ & 0.004 \\
\hline $\mathrm{G} 2+\mathrm{G} 3$ & 74 & $16(22)$ & $58(78)$ & \\
\hline \multicolumn{5}{|l|}{ T stage } \\
\hline Tis $+\mathrm{T} 1$ & 14 & $7(50)$ & $7(50)$ & 0.000 \\
\hline $\mathrm{T} 2$ & 68 & $29(43)$ & 39 (57) & \\
\hline T3 & 83 & $19(23)$ & $64(77)$ & \\
\hline \multicolumn{5}{|c|}{ Lymph node metastasis } \\
\hline Presence & 67 & 13 (19) & $54(81)$ & 0.002 \\
\hline Absence & 98 & $42(43)$ & $56(57)$ & \\
\hline \multicolumn{5}{|l|}{ UICC stage } \\
\hline $0+\mathrm{I}$ & 48 & $27(56)$ & $21(44)$ & 0.000 \\
\hline II & 73 & $20(27)$ & $53(73)$ & \\
\hline III & 44 & 8 (18) & $36(82)$ & \\
\hline
\end{tabular}

Abbreviations: G1, G2 and G3, tumor histological grades I, II and III, respectively; Tis, carcinoma in situ.

$P<0.05$; Figure $3 \mathrm{~d}$ ). These results indicate that ALDH1A1 expression is correlated with the development and progression of esophageal squamous cell carcinoma.

\section{High ALDH1A1 Expression is Correlated with Poor Patient Prognosis}

We then analyzed the relationship between ALDH1A1 expression and the survival of a total 134 esophageal squamous cell carcinoma patients with follow-up data. Median survival time of the total patients was 31 months (ranging from 1 to more than 75 months). Kaplan-Meier survival curves showed that the 5-year survival rates for patients with ALDH1A1 1ow tumors were $46 \%$, but $23 \%$ for patients with ALDH1A1 $1^{\text {high }}$ tumors (Figure 4a, $P=0.008)$. Patients with ALDH1A1 low tumors at early stages (IA $+\mathrm{IB}$ ) (Figure $4 \mathrm{~b}, P=0.02$ ) or with well-differentiated esophageal squamous cell carcinoma (G1) (Figure 4c, $P=0.021$ ) had longer lifespan than those with ALDH1A1 $1^{\text {high }}$ tumors. However, the survival rate of patients with later stage (II and III) $(P>0.05)$ or moderately and poorly differentiated esophageal squamous cell carcinoma (G2 and 3) $(P>0.05)$ were similar, regardless of ALDH1A1positivity. Thus, ALDH1A1 predicts esophageal squamous cell carcinoma outcome and can be used as a biomarker for patient prognosis.

\section{Discussion}

In this study, we revealed for the first time that ALDH1A1 $1^{\text {high }}$ esophageal squamous cell carcinoma cells possess cancer stem cell property and that the expression of ALDH1A1 is associated with esophageal squamous dysplasia and carcinoma. Esophageal squamous dysplasia, a precancerous lesion of esophageal squamous cell carcinoma, refers to abnormal morphological changes of squamous cells. Thus, ALDH1A1-expressing cells have a crucial role in the initiation and progression of esophageal squamous cell carcinoma.

ALDH1A1 is a predominant isoform of aldehyde dehydrogenase family in mammals located in the cytoplasm $^{15,16}$ and is originally considered as a marker for normal hematopoietic progenitor cells, ${ }^{17}$ and is also found in cancer cells with stem cell property, that is, cancer stem-like cells, in several malignant tumors including leukemia, ${ }^{9}$ breast cancer $^{7}$ and lung cancer. ${ }^{8,18}$ Wang et $a 1^{19}$ reported that ALDH1A1 was located in the nuclei of esophageal squamous cell carcinoma cells. However, we detected that ALDH1A1 protein mainly in the cytoplasm of precancerous and cancer cells of esophagus, consistent with reports on breast and ovarian tumors. ${ }^{7,20}$ Our data showed that in the low-grade esophageal squamous dysplasia, ALDH1A1-positive cells were distributed in the basal layer of the mucosa, suggesting normal mucosal stem cells as one of the sources for cancer stem-like cells. ${ }^{21}$ Further, ALDH1A1 expression was positively correlated with the UICC stages, invasion depth and lymph node metastasis of esophageal squamous cell carcinoma and was associated with shorter survival of the patients. Therefore, ALDH1A1-expressing cells are crucial for the development and progression of esophageal squamous cell carcinoma and ALDH1A1 may be used as a biomarker for malignancy of esophageal squamous cell carcinoma and a predictor of patient prognosis.

It is important to note that ALDH1A1 ${ }^{\text {high }}$ cells possess the property of cancer stem-like cells in esophageal squamous cell carcinoma. It has been reported that esophageal cancer stem-like cells may reside in a side population of cancer cells. ${ }^{22-24}$ However, Zhao et al25 found no correlation between the presence of side population of cancer cells and the tumorigenicity of the esophageal squamous cell carcinoma cells. CD44 has also been proposed as a marker for cancer stem-like cells in esophageal squamous cell carcinoma, although most of the esophageal squamous cell carcinoma cells express CD44. ${ }^{25,26}$ Another molecule, CD90 as a cancer stem-like cell marker of esophageal squamous cell carcinoma cells, remains debatable. ${ }^{25,27}$ Tumorsphere culture and pretreatment with radiation may be used for enrichment of cancer stem-like cells from esophageal squamous cell carcinoma; however, the cell heterogeneity limits 

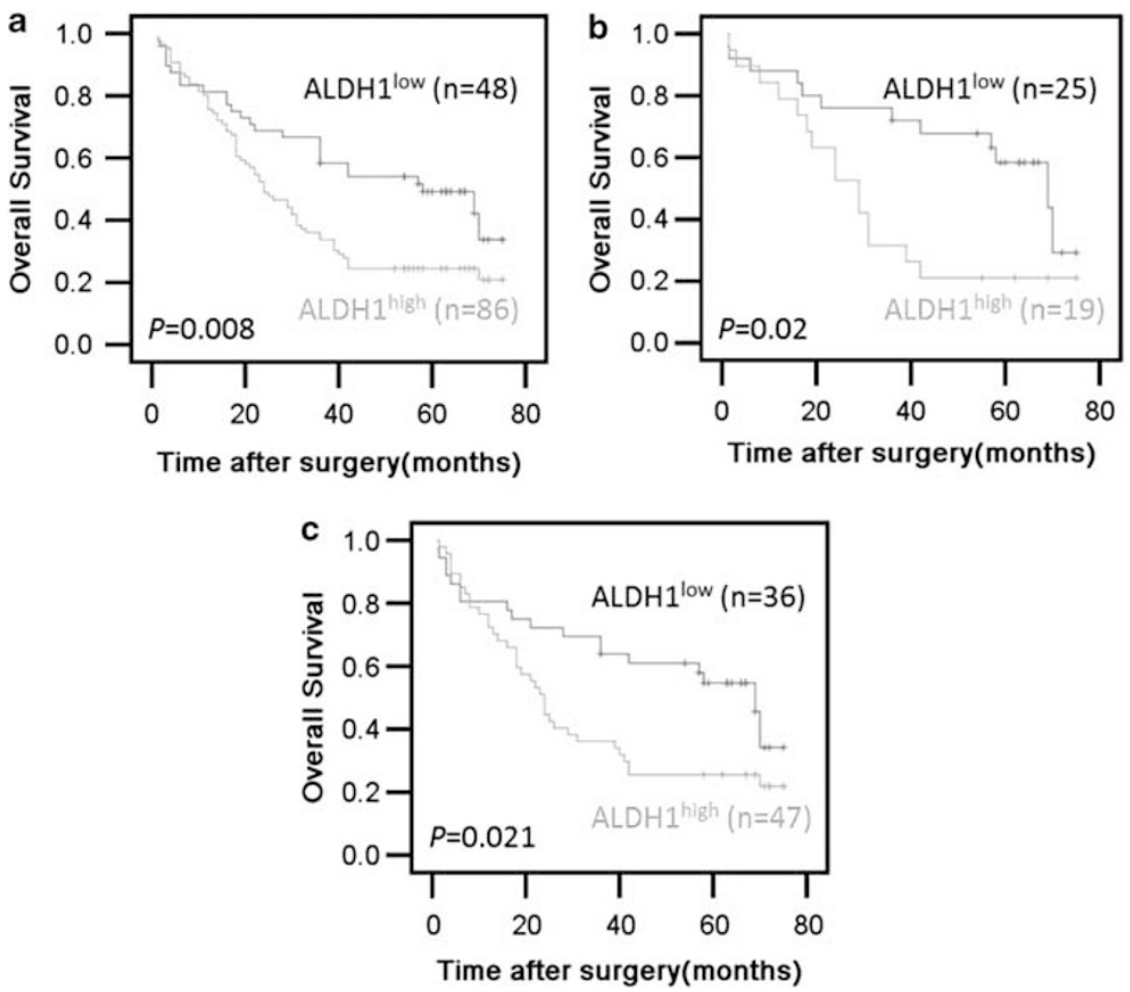

Figure 4 Analysis of correlation between overall patient survival rates and ALDH1A1 expression in the specimens from esophageal squamous cell carcinoma patients by Kaplan-Meier estimation. (a) Kaplan-Meier estimation performed on all of the esophageal squamous cell carcinoma patients indicates that the patients with ALDH1A1 ${ }^{\text {high }}$ expression have shorter overall survival time than the patients with $A L D H 1 A 1^{\text {low }}$ expression $\left(A L D H 1 A 1^{\text {low }}=48\right.$ and $\left.A L D H 1 A 1^{\text {high }}=86\right)$. (b) Kaplan-Meier estimation performed on the esophageal squamous cell carcinoma patients at stage I indicates that the patients with ALDH1A1 high expression have shorter overall survival time than the patients with ALDH1A1 low expression (ALDH1A1 1ow, $n=25$ and ALDH1A1 high, $n=19$ ). (c) Kaplan-Meier estimation performed on the esophageal squamous cell carcinoma patients at G1 grade indicates that the patients with ALDH1A1 high expression have shorter overall survival time than the patients with ALDH1A $1^{\text {low }}$ expression (ALDH1A1 ${ }^{\text {low }}, n=36$ and ALDH1A1 ${ }^{\text {high }}$, $n=47$ ). ALDH1A1, aldehyde dehydrogenase 1A1.

the application. ${ }^{28-30}$ Recently, ALDH1A1 was found increased in tumor sphere ${ }^{29}$ and three-dimensional cultured cancer stem-like cells ${ }^{31}$ of esophageal squamous cell carcinoma cells, suggesting that ALDH1A1 might be a better biomarker for the cancer stem-like cells of esophageal squamous cell carcinoma. Our study shows that ALDH1A1 $1^{\text {high }}$ cells have capabilities of self-renewal, differentiation and tumor initiation, thereby representing as a more reliable marker for the identification and isolation of cancer stem-like cells in esophageal squamous cell carcinoma.

Cancer stem-like cells are involved in the invasion and metastasis with yet to be defined mechanisms. We found that ALDH1A1-positive cells were preferentially distributed in the invasion frontier of esophageal squamous cell carcinoma and metastatic lesions. Our in vitro results also demonstrated that ALDH1A1 $1^{\text {high }}$ esophageal squamous cell carcinoma cells were more highly invasive and metastatic. ALDH1A1 ${ }^{\text {high }}$ cancer stem-like cells also express high levels of vimentin but low levels of E-cadherin, indicating an epithelial-mesenchymal transition phenotype of ALDH1A1 ${ }^{\text {high }}$ cancer stem-like cells. ${ }^{32}$ MMP family members are capable of degrading extracellular matrix proteins and pave a path for cancer cell migration and invasion. ${ }^{33}$ Thus, ALDH1A1 $1^{\text {high }}$ cancer stem-like cells, by expressing high levels of MMPs and exhibiting an epithelialmesenchymal transition phenotype, contribute to the invasion and metastasis of esophageal squamous cell carcinoma. In summary, we provide the strong evidence that ALDH1A 1 high cancer stem-like cells contribute to the invasion, metastasis and poor outcome of human esophageal squamous cell carcinoma.

\section{Acknowledgments}

We thank Dr Ji Ming Wang, Laboratory of Molecular Immunoregulation, Cancer and Inflammation Program, Center for Cancer Research, National Cancer Institute, USA, for his critical review. We thank Qing-hua Ma and Zeng-tian Sun for FACS cell sorting and Zhi-cheng He, Hui Zeng and Jie Li, Institute of Pathology and Southwest Cancer Center, Southwest Hospital, Third Military Medical University, for their technical assistance. This study was supported by grants from the National Basic Research Program of China (973 Program, No. 2010CB529403) and the National Natural Science 
Foundation of China (NSFC Nos. 81071771, 30725035 and 30930103).

\section{Disclosure/conflict of interest}

The authors declare no conflict of interest.

\section{References}

1 Zhang HZ, Jin GF, Shen HB. Epidemiologic differences in esophageal cancer between Asian and Western populations. Chin J Cancer 2012;31:281-286.

2 Jemal A, Bray F, Center MM, et al. Global cancer statistics. CA Cancer J Clin 2011;61:69-90.

3 Clarke MF, Dick JE, Dirks PB, et al. Cancer stem cellsperspectives on current status and future directions: AACR Workshop on cancer stem cells. Cancer Res 2006;66:9339-9344.

4 Yang L, Ping YF, Yu X, et al. Gastric cancer stem-like cells possess higher capability of invasion and metastasis in association with a mesenchymal transition phenotype. Cancer Lett 2011;310:46-52.

$5 \mathrm{Yu}$ SC, Bian XW. Enrichment of cancer stem cells based on heterogeneity of invasiveness. Stem Cell Rev 2009;5:66-71.

$6 \mathrm{Ye} \mathrm{XZ}, \mathrm{Xu}$ SL, Xin YH, et al. Tumor-associated microglia/macrophages enhance the invasion of glioma stem-like cells via TGF-beta1 signaling pathway. J Immunol 2012;189:444-453.

7 Ginestier C, Hur MH, Charafe-Jauffret E, et al. ALDH1A1 is a marker of normal and malignant human mammary stem cells and a predictor of poor clinical outcome. Cell Stem Cell 2007;1:555-567.

8 Huang CP, Tsai MF, Chang TH, et al. ALDH-positive lung cancer stem cells confer resistance to epidermal growth factor receptor tyrosine kinase inhibitors. Cancer Lett 2013;328:144-151.

9 Fleischman AG. ALDH marks leukemia stem cell. Blood 2012;119:3376-3377.

10 Kunju LP, Cookingham C, Toy KA, et al. EZH2 and ALDH-1 mark breast epithelium at risk for breast cancer development. Mod Pathol 2011;24:786-793.

11 Rota LM, Lazzarino DA, Ziegler AN, et al. Determining mammosphere-forming potential: application of the limiting dilution analysis. J Mammary Gland Biol Neoplasia 2012;17:119-123.

12 Oskarsson $\mathrm{T}$, Acharyya S, Zhang $\mathrm{XH}$, et al. Breast cancer cells produce tenascin $\mathrm{C}$ as a metastatic niche component to colonize the lungs. Nat Med 2011;17: 867-874.

13 Ito E, Ozawa S, Kijima H, et al. New invasive patterns as a prognostic factor for superficial esophageal cancer. J Gastroenterol 2012;47:1279-1289.

14 Talsma K, van Hagen P, Grotenhuis BA, et al. Comparison of the 6th and 7th Editions of the UICC-AJCC TNM Classification for Esophageal Cancer. Ann Surg Oncol 2012;19:2142-2148.

15 Hess DA, Wirthlin L, Craft TP, et al. Selection based on CD133 and high aldehyde dehydrogenase activity isolates long-term reconstituting human hematopoietic stem cells. Blood 2006;107:2162-2169.
16 Hess DA, Craft TP, Wirthlin L, et al. Widespread nonhematopoietic tissue distribution by transplanted human progenitor cells with high aldehyde dehydrogenase activity. Stem Cells 2008;26:611-620.

17 Storms RW, Trujillo AP, Springer JB, et al. Isolation of primitive human hematopoietic progenitors on the basis of aldehyde dehydrogenase activity. Proc Natl Acad Sci USA 1999;96:9118-9123.

18 Jiang F, Qiu Q, Khanna A, et al. Aldehyde dehydrogenase 1 is a tumor stem cell-associated marker in lung cancer. Mol Cancer Res 2009;7:330-338.

19 Wang Y, Zhe H, Gao P, et al. Cancer stem cell marker ALDH1A1 expression is associated with lymph node metastasis and poor survival in esophageal squamous cell carcinoma: a study from high incidence area of northern China. Dis Esophagus 2012;25:560-565.

20 Chang B, Liu G, Xue F, et al. ALDH1A1 expression correlates with favorable prognosis in ovarian cancers. Mod Pathol 2009;22:817-823.

21 Reya T, Morrison SJ, Clarke MF, et al. Stem cells, cancer, and cancer stem cells. Nature 2001;414:105-111.

22 Huang D, Gao Q, Guo L, et al. Isolation and identification of cancer stem-like cells in esophageal carcinoma cell lines. Stem Cells Dev 2009;18:465-473.

23 Chen Y, Li D, Wang D, et al. Quiescence and attenuated DNA damage response promote survival of esophageal cancer stem cells. J Cell Biochem 2012;113:3643-3652.

$24 \mathrm{Li} \mathrm{H}$, Gao Q, Guo L, et al. The PTEN/PI3K/Akt pathway regulates stem-like cells in primary esophageal carcinoma cells. Cancer Biol Ther 2011;11:950-958.

25 Zhao JS, Li WJ, Ge D, et al. Tumor initiating cells in esophageal squamous cell carcinomas express high levels of CD44. PLoS One 2011;6:e21419.

26 Rassouli FB, Matin MM, Bahrami AR, et al. Evaluating stem and cancerous biomarkers in CD15CD44 KYSE30 cells. Tumour Biol 2013; PMID: 23797812 (e-pub ahead of print).

27 Tang KH, Dai YD, Tong M, et al. A CD90(+) tumorinitiating cell population with an aggressive signature and metastatic capacity in esophageal cancer. Cancer Res 2013;73:2322-2332.

28 Zhao R, Quaroni L, Casson AG. Identification and characterization of stemlike cells in human esophageal adenocarcinoma and normal epithelial cell lines. J Thorac Cardiovasc Surg 2012;144:1192-1199.

29 Zhang G, Ma L, Xie YK, et al. Esophageal cancer tumorspheres involve cancer stem-like populations with elevated aldehyde dehydrogenase enzymatic activity. Mol Med Rep 2012;6:519-524.

30 Zhang X, Komaki R, Wang L, et al. Treatment of radioresistant stem-like esophageal cancer cells by an apoptotic gene-armed, telomerase-specific oncolytic adenovirus. Clin Cancer Res 2008;14:2813-2823.

31 Fujiwara D, Kato K, Nohara S, et al. The usefulness of three-dimensional cell culture in induction of cancer stem cells from esophageal squamous cell carcinoma cell lines. Biochem Biophys Res Commun 2013;434:773-778.

32 Yang J, Weomberg RA. Epithelial-mesenchymal transition: at the crossroads of development and tumor metastasis. Dev Cell 2008;14:818-829.

33 Roy R, Yang J, Moses MA. Matrix metalloproteinases as novel biomarkers and potential therapeutic targets in human cancer. J Clin Oncol 2009;27:5287-5297. 